\title{
The latency for correcting a movement depends on the visual attribute that defines the target
}

\author{
Margot M. Veerman · Eli Brenner · Jeroen B. J. Smeets
}

Received: 20 October 2007 / Accepted: 19 January 2008 / Published online: 7 February 2008

(C) The Author(s) 2008

\begin{abstract}
Neurons in different cortical visual areas respond to different visual attributes with different latencies. How does this affect the on-line control of our actions? We studied hand movements directed toward targets that could be distinguished from other objects by luminance, size, orientation, color, shape or texture. In some trials, the target changed places with one of the other objects at the onset of the hand's movement. We determined the latency for correcting the movement of the hand in the direction of the new target location. We show that subjects can correct their movements at short latency for all attributes, but that responses for the attributes color, form and texture (that are relevant for recognizing the object) are $50 \mathrm{~ms}$ slower than for the attributes luminance, orientation and size. This dichotomy corresponds to both to the distinction between magno-cellular and parvo-cellular pathways and to a dorsal-ventral distinction. The latency also differed systematically between subjects, independent of their reaction time.
\end{abstract}

Keywords Dorsal stream · Hand movement · Color . Shape $\cdot$ Visual processing $\cdot$ Target perturbation .

Double step

M. M. Veerman

Department of Neuroscience, Erasmus MC,

Rotterdam, The Netherlands

M. M. Veerman · E. Brenner · J. B. J. Smeets $(\bowtie)$ Research Institute MOVE, Faculty of Human Movement Sciences, VU University Amsterdam, van der Boechorststraat 9, 1081 BT Amsterdam, The Netherlands

e-mail: J.Smeets@fbw.vu.nl

\section{Introduction}

Neurons in different cortical visual areas respond to different visual attributes with different latencies (Livingstone and Hubel 1987, 1988; Schmolesky et al. 1998; Bullier 2001). How do such differences affect the way in which visual information guides our movements? Two main streams of visual processing have been identified within the brain; each specialized for processing a different kind of information (Trevarthen 1968; Mishkin et al. 1983; Goodale and Milner 1992; Ungerleider and Haxby 1994). A ventral stream in which information travels from V1 to the inferotemporal cortex is crucial for the identification of objects, whereas a dorsal stream passing through the posterior parietal region plays a major role in spatial vision. A widely accepted explanation for this distinction is that the dorsal stream is specialized for quickly processing spatial information to control action, whereas the slower, detailed visual analysis of other attributes that are important for recognizing objects takes place in the ventral pathway (DeYoe and Van Essen 1988; Goodale and Milner 1992; Tanne et al. 1995; Desmurget et al. 1999; Rossetti et al. 2000; Lee and van Donkelaar 2002). This view implies that not all information can be used to quickly adjust hand movements. We tested this prediction.

One of the problems with comparing experiments that investigate whether various kinds of visual information can contribute to fast control of actions is that investigators use different experimental paradigms for different visual attributes, which themselves may give different results for a single attribute (e.g., compare Pisella et al. 1998; Schmidt 2002; Brenner and Smeets 2004; Cressman et al. 2006; White et al. 2006 for the role of color in guiding hand movements). It would therefore be very useful to systematically investigate the latency of responses to various visual 
attributes with a single paradigm. We therefore compared responses to changes in target location for hand movements directed toward targets defined by attributes that are normally relevant for goal-directed actions (luminance-contrast, size and orientation) with ones toward targets defined by attributes that are normally more relevant for object recognition (color, shape and texture). In all cases, there were three possible target positions. The target was at one of them. Other objects (references) occupied the other two positions. In half of the trials, the target switched locations with one of the references just after the hand started moving (Fig. 1a, b).

An issue that cannot be ignored is how conspicuous the distinction between target and reference is within each attribute; i.e., how easily the target can be distinguished from the references. When comparing responses to targets defined by the same attribute, the response becomes faster if the relevant contrast is increased (Brenner and Smeets 2003). We therefore have to show that any differences in latency that we find cannot be simply caused by differences in conspicuousness. The question is how to evaluate conspicuousness independently from the response latency.

In previous studies, this issue was resolved by equating a critical stimulus parameter (e.g. cone contrast, White et al. 2006), or by equating the stimuli in terms of an independent perceptual judgment (e.g., how conspicuous the targets are judged to be, Brenner and Smeets 2003). The first approach is not feasible in our current experiment because it is not at all clear how stimulus parameters for different attributes could be matched. The second approach is also problematic, because the visual processing for the perceptual

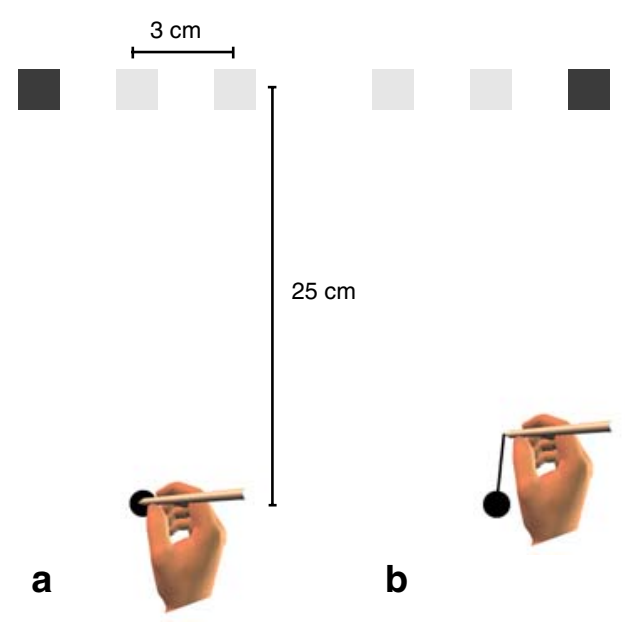

Fig. 1 Schematic overview of the experiment. a The participant holds a pen at the starting position when a target (dark square in this example) and two other objects (references, the brighter squares in this example) appeared. b In half of the trials, the target switches to one of the other locations just after movement onset. $\mathbf{c}$ Ten paths of one judgment task might differ from that for the real experiment. We will therefore develop a new method to deal with differences in conspicuousness. This method, which will be developed in the results section, is based on examining responses to various targets that are all distinguished on the basis of the same attribute, but that differ in conspicuousness. We will show that beside leading to shorter latencies, more conspicuous targets also lead to shorter reaction times, higher velocities, and a steeper slope of the responses. We will then compare latencies for targets defined by different attributes when they give rise to comparable reaction times, velocities, and response slopes. Differences in latency after such matching cannot be caused by differences in conspicuousness.

\section{Materials and methods}

\section{Participants}

Twelve subjects took part in this study. Three of them were the authors and the others were colleagues and friends who were unaware of the hypothesis that was being tested. All of them had normal or corrected to normal vision. Two of the participants were left-handed. Each participant made 120 pointing movements in each of 13 different sessions. Six of the participants also made 120 pointing movements in each of 7 additional sessions in which various luminance contrasts were used. Each participant performed the sessions in a different random order. The ethics committee of the Faculty of Human Movement Sciences approved the study.

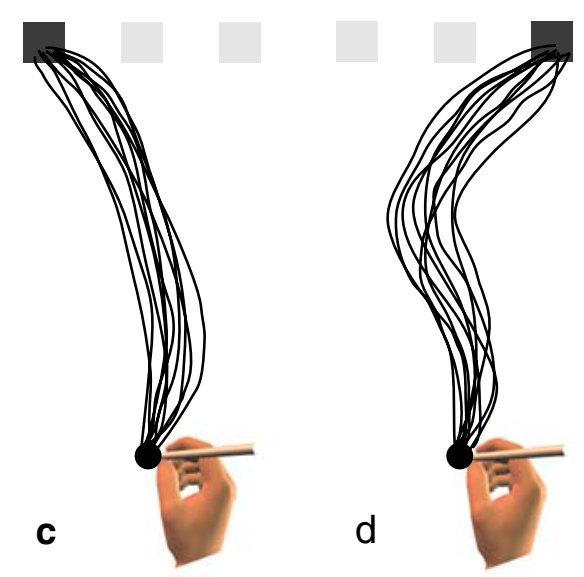

participant's hand (P6) for a target that remained at the leftmost position in the "luminance (390\%)" condition. d All ten paths of the same participant's hand in the condition in which the target jumped from the leftmost to the rightmost position at movement onset 
Apparatus and stimuli

Participants sat behind an A2-sized graphic tablet (Digitizer II, Wacom Ltd, Tokyo, Japan) and viewed a projection surface via a semitransparent mirror that was placed above this graphic tablet. The images were back-projected from above the projection surface. The distance between the mirror and the projection surface was identical to that between the mirror and the surface of the tablet, so that the projected image appeared to be at the surface of the tablet. Lamps underneath the mirror ensured that the participants could see their hands. The resolution of the projected image was 1,024 by 768 pixels, with 1 pixel corresponding to about $0.5 \mathrm{~mm}$. The position of the pen was determined every $5 \mathrm{~ms}$ $(200 \mathrm{~Hz})$.

At the near edge of the screen was a starting point (a black, $1 \mathrm{~cm}$ diameter disk with a luminance of $2.3 \mathrm{~cd} / \mathrm{m}^{2}$ as measured with a Minolta LS-110 luminance meter). At a distance of $25 \mathrm{~cm}$ from this point in the sagittal direction, three objects were projected next to each other on a white background $\left(35 \times 45 \mathrm{~cm} ; 28.0 \mathrm{~cd} / \mathrm{m}^{2}\right)$. The distance between the centers of the objects was $3 \mathrm{~cm}$ (Fig. 1a). One of the three objects served as the target; this object differed from the other two objects (the references).

We varied the attribute in which the target differed from the references (and the magnitude of this difference) between conditions (Fig. 2). Each condition was tested in a separate session. In the control condition, the reference objects were equal to the background so they were invisible: only the dark gray target square $\left(1 \mathrm{~cm}\right.$ sides, $\left.5.1 \mathrm{~cd} / \mathrm{m}^{2}\right)$ was visible.

In the luminance $(390 \%)$ condition, the target differed from the references in luminance only (for further details about this and subsequent conditions see Fig. 2). There were seven additional conditions with different luminance contrasts between the target and the references. These were used to determine how responses depend on how conspicuous the distinction is between the target and the references.

In the three orientation conditions, the target and the references were rectangles $\left(0.67 \times 1.50 \mathrm{~cm}, 5.1 \mathrm{~cd} / \mathrm{m}^{2}\right)$. The target rectangle was orientated at an angle of $45^{\circ}, 60^{\circ}$ or $90^{\circ}$ with respect to the parallel references. In the three size conditions the target squares had different sizes. The reference squares had surface areas of $1 \mathrm{~cm}^{2}$, as did all the objects in the other conditions. In the shape session the target was a circle, and the references were squares. In the color condition, the target was a green square $\left(5.1 \mathrm{~cd} / \mathrm{m}^{2}\right)$ and the references were two red squares $\left(5.1 \mathrm{~cd} / \mathrm{m}^{2}\right)$. In the texture condition, the target square was filled with ten 1$\mathrm{mm}$ wide stripes, alternating between dark $\left(2.3 \mathrm{~cd} / \mathrm{m}^{2}\right)$ and bright $\left(7.9 \mathrm{~cd} / \mathrm{m}^{2}\right)$ ones. The average luminance of the stripes was equal to the luminance of the references $(5.1 \mathrm{~cd} /$ $\mathrm{m}^{2}$ ), to ensure that participants had to analyze the texture to recognize the target.

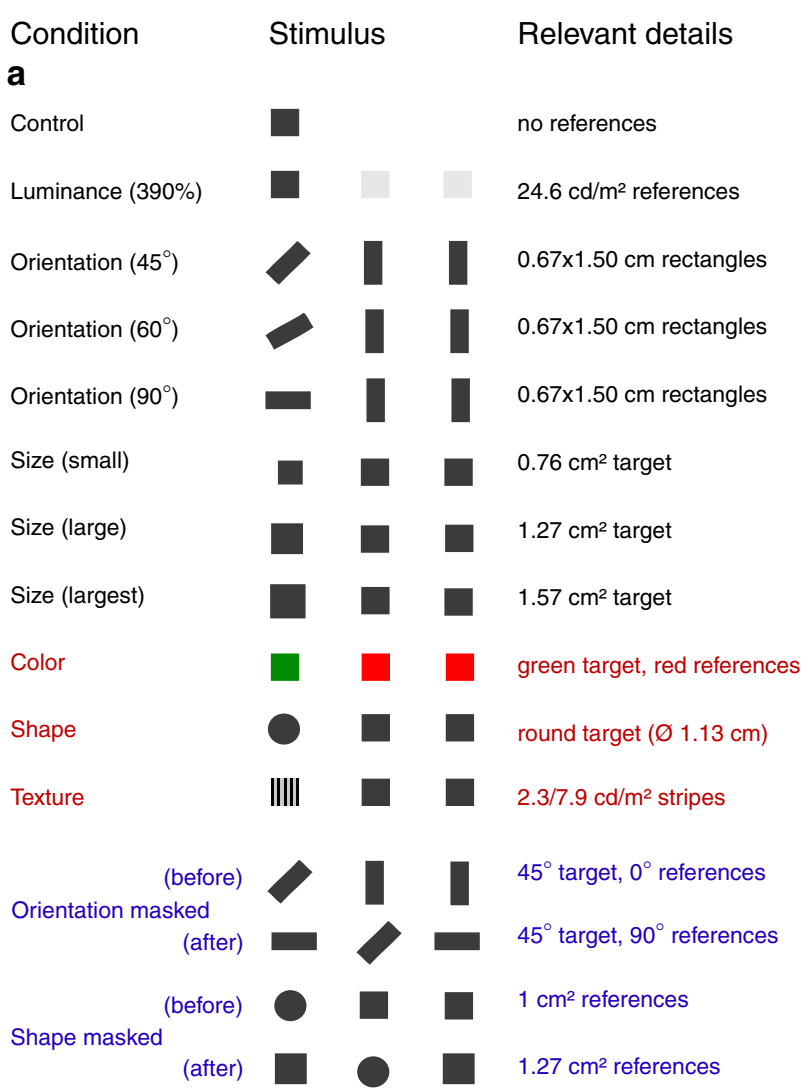

b

Luminance (160\%)

Luminance (140\%)

Luminance (120\%)

Luminance (100\%)

Luminance (80\%)

Luminance $(60 \%)$

Luminance $(40 \%)$

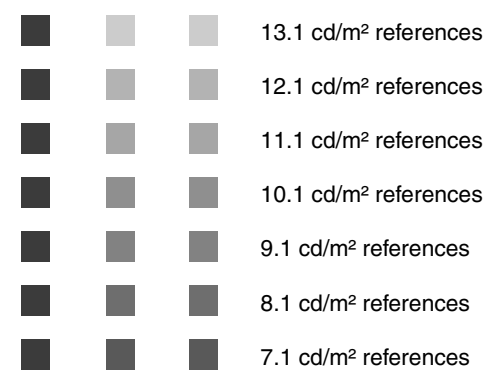

Fig. 2 Schematic representations of the stimuli in the 20 conditions (depicted with the target on the left). The luminance of both the target and the references is $5.1 \mathrm{~cd} / \mathrm{m}^{2}$ and they are both $1 \mathrm{~cm}^{2}$ gray squares unless otherwise mentioned (see "relevant details"). a The 13 conditions that were performed by all participants. Red lettering indicates that the responses depend on attributes that are normally needed to recognize objects, and blue lettering indicates that all items change. b The seven additional luminance conditions that were performed by six participants (numbers between brackets are the percentage that the references are brighter than the target)

During pilot-experiments, some of the participants had the impression (especially in the orientation and the shape conditions) that they were not responding to a difference between the target and the references, but to the change at the new target position. To investigate whether this was really an issue we performed two control conditions in which we masked such effects by also changing aspects of the references when the target changed its position. In a 
masked orientation condition, the references both changed their orientation by $90^{\circ}$ when the target jumped to a new location. In a masked shape condition, the reference squares increased in size at the same moment that the target circle changed position.

\section{Procedure}

Participants started each trial by placing the pen on the starting point. After a random time (about $2 \mathrm{~s}$ ), the target and references appeared (Fig. 1a). The task was to reach the target with the pen and stop there as soon as possible. The sum of the movement time and the reaction time was to be minimized. Participants were shown which object was the target (and which the references) before they started each session.

Within each session of 120 trials, the target appeared 40 times at each of the 3 positions. In half of the trials the target and the references remained at their initial position (unperturbed trials, Fig. 1c). In the other half of the trials the target changed its position to one of the two reference positions about $62 \mathrm{~ms}$ after the subject's finger reached a velocity threshold of $0.02 \mathrm{~m} / \mathrm{s}$ (perturbed trials, Fig. 1b). At the same moment the reference (that had been at that position) changed its position to where the target had been. Whenever the target changed position participants had to adjust their hand movement to reach the new target location (Fig. 1d). If a movement ended on the target within $1.2 \mathrm{~s}$ of the target first appearing, the participant was rewarded with auditory feedback. The 120 trials (three initial positions, change in position or not, two positions to which to change, ten replications) were presented in random order. Since different positions to which to change are irrelevant when there is no change, there were actually 20 trials in which the target remained at each initial position.

\section{Analysis}

Velocities were calculated for the interval between every two measurements by dividing the displacement of the tip of the pen by the $5 \mathrm{~ms}$ between the measurements. The beginning of the movement was defined as the first position after the tangential velocity reached $0.02 \mathrm{~m} / \mathrm{s}$. The end of the movement was defined as the first position after the tangential velocity fell below $0.02 \mathrm{~m} / \mathrm{s}$. To evaluate the corrections, we only used the lateral component of the velocity (parallel to the displacement of the target).

In order to isolate the responses to changes in target position, we first synchronized all the measurements relative to the moment that the target changed - or would have changed-position. We then separately averaged the lateral velocity for each combination of initial and final target position. We defined the lateral velocity in the direction of the position change as being positive; when the position did not change, we defined the direction in which it would have changed as being positive. We characterize the response to a change in target position by the additional lateral velocity: the difference in the lateral component of the velocity between the trials in which the target changed from a certain initial position to a certain final position and the ones in which it remained at the same initial position. To obtain a single response per attribute for each participant we averaged the additional lateral velocity across the six combinations of initial and final target positions. We used these curves to determine the latencies of the responses.

To estimate the latencies of the responses we approximated the initial responses by straight lines (dotted lines in Fig. 3). We used a robust method (one that is not too sensitive to the exact shape of the response near its onset) to approximate the initial response: we determined the amplitude of the peak in the average additional lateral velocity and then determined the points at which the additional lateral velocity reached 25 and $75 \%$ of this value. We consider a line through these two points as a good and robust approximation of the initial response. We considered the intersection of this line with a line at an additional lateral velocity of zero to give a reliable estimate of the latency of the reaction to the change in target position. We evaluated the influence of the conditions on the slopes of the abovementioned lines and on their intersection points with the zero-velocity line (across and participants) using paired $t$ tests.

The slope of the line through the 25 and $75 \%$ points of the average additional lateral velocity does not only depend on the intensity of the response on individual trials (lower acceleration results in a shallower slope). If the response does not always occur at the same time, averaging will result in an average response that has a lower peak velocity, shallower slope and longer duration than the responses in individual trials. To evaluate the shape of the response curve without the influence of variability in timing, we used a second way of synchronizing trials before determining the additional lateral velocity.

To estimate the intensity of individual responses for each condition and participant, we synchronized the lateral velocity curves of all perturbed trials at the peak lateral velocity in the direction of the new target position (irrespective of when the target position changed), and produced an additional lateral velocity curve for each subject by averaging across replications and perturbation directions. We averaged these synchronized additional lateral velocity curves across positions and across participants to investigate whether there are systematic differences between the intensity of the responses for different attributes. We averaged the curves across conditions rather than participants to investigate whether there are systematic differences between the intensity of different participants' 

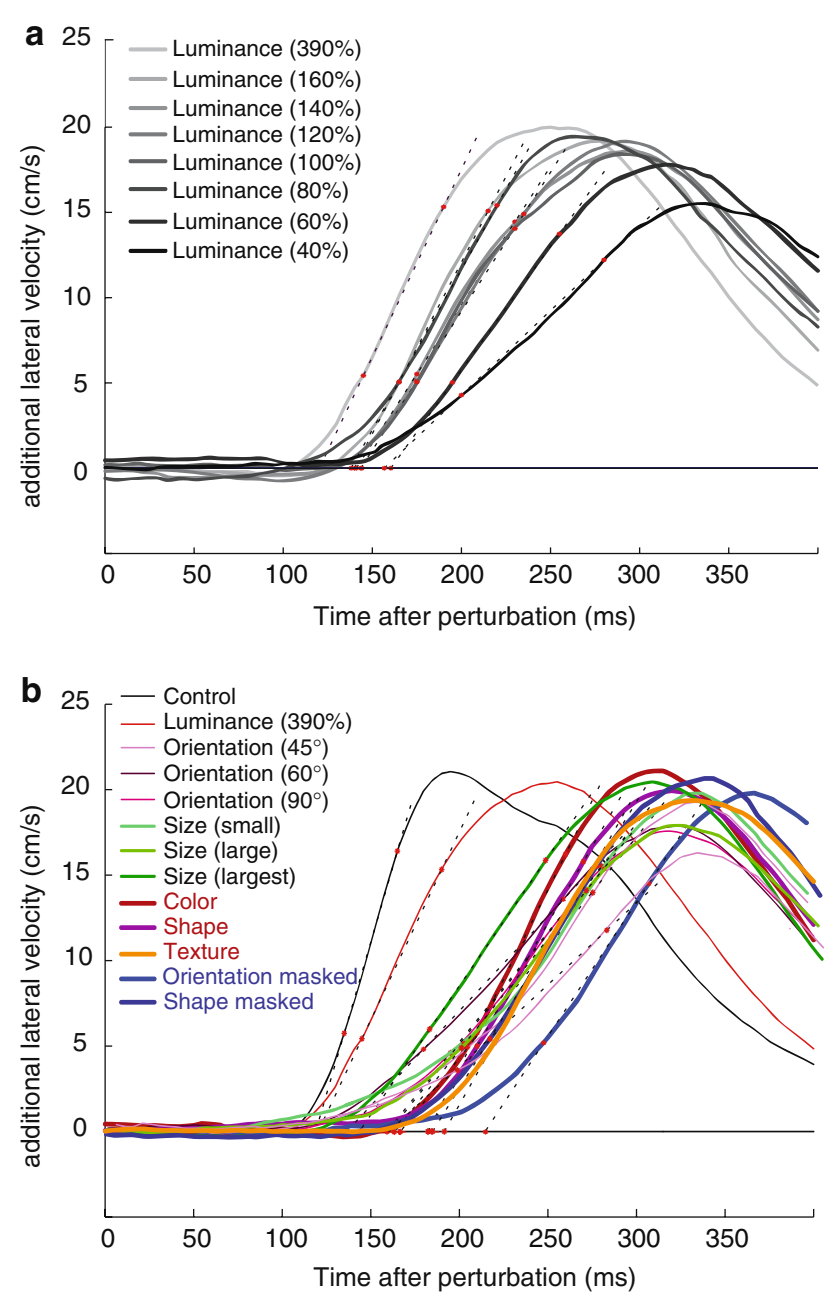

Fig. 3 Mean additional lateral velocity for each attribute as a function of the time after the perturbation. The mean additional lateral velocity is the mean difference between the lateral velocity on the trials in which the target did and did not change position. To estimate the latency of the response, a line through the points at 25 and $75 \%$ of the peak velocity (see dotted lines) was extrapolated to intersect the line representing an additional velocity of 0 (black horizontal line). The time at the point of intersection was considered to be the response latency. a Averages of six participants for the luminance contrast conditions. b Averages of 12 participants for the conditions in which they all participated

responses. In order to determine whether the response was proportional to the size of the perturbation, we also averaged the curves separately for all pairs of positions in which the target jumped $3 \mathrm{~cm}$, and all pairs of positions in which the target jumped $6 \mathrm{~cm}$.

\section{Results}

\section{Overview of responses}

On average, it took participants $365 \mathrm{~ms}$ to react to the appearance of the target (and the two references). The average movement time (time until participants stopped their movement on the target) was another $275 \mathrm{~ms}$. Figure 1 shows one participant's paths in one condition with the target initially positioned on the left. When the target stayed at the initial target position (Fig. 1c), the hand moved to the target along a slightly curved path (note that the lateral deviations are exaggerated due to the unequal scaling of the figure). When the target jumped at the onset of the movement (Fig. 1d), participants obviously initially moved along similar paths to those seen in Fig. 1c, but approximately half way to the target the path curved toward the new target position.

Figure 3 shows the additional lateral velocity for the various conditions (summarized in Fig. 2), averaged over all subjects. Each curve represents the difference in lateral velocity between trials in which the target did and did not change position (see "materials and methods" for details). The dotted lines are drawn through the points at which the mean additional lateral velocity curves reached 25 and $75 \%$ of their peak values. We consider the points at which these lines intersected the line at an additional lateral velocity of zero as the latencies of the responses. We used targets that only differed in luminance from the references to evaluate effects of conspicuousness (for half of our subjects). We found that lower luminance contrast (a less conspicuous target) results in both a longer latency and a shallower slope (Fig. 3a). The conclusion that we can draw from the main conditions depicted in Fig. 3b, is that both the timing and the slopes of the curves differ between conditions. Figure 3 shows an analysis over responses averaged over subjects. The rest of the analysis is done on the basis of determining slopes and latencies for each subject (and condition) separately.

Dealing with conspicuousness

It is clear from Fig. 3a that the latencies are larger for less conspicuous targets. In order to compare the latencies of responses to targets defined by different attributes, we have to take into account differences in conspicuousness. Figure $4 a, b$ and $c$ shows how not only the latency of the response varies with conspicuousness, but also the reaction time of the initial movements toward less conspicuous targets are longer (Fig. 4a), the slope of the response to a perturbation is shallower (Fig. 4b), and both the peak velocity of the initial movement toward the target and that of the correction are slower (Fig. 4c). All three plots yield significant correlations $(P<0.05)$, and the data are distributed along the fitted line with deviations that can be expected on the basis of the error bars.

Since several measures co-vary with the conspicuousness (i.e. the luminance contrast) of the target, we can use these measures to evaluate whether latency differences can 

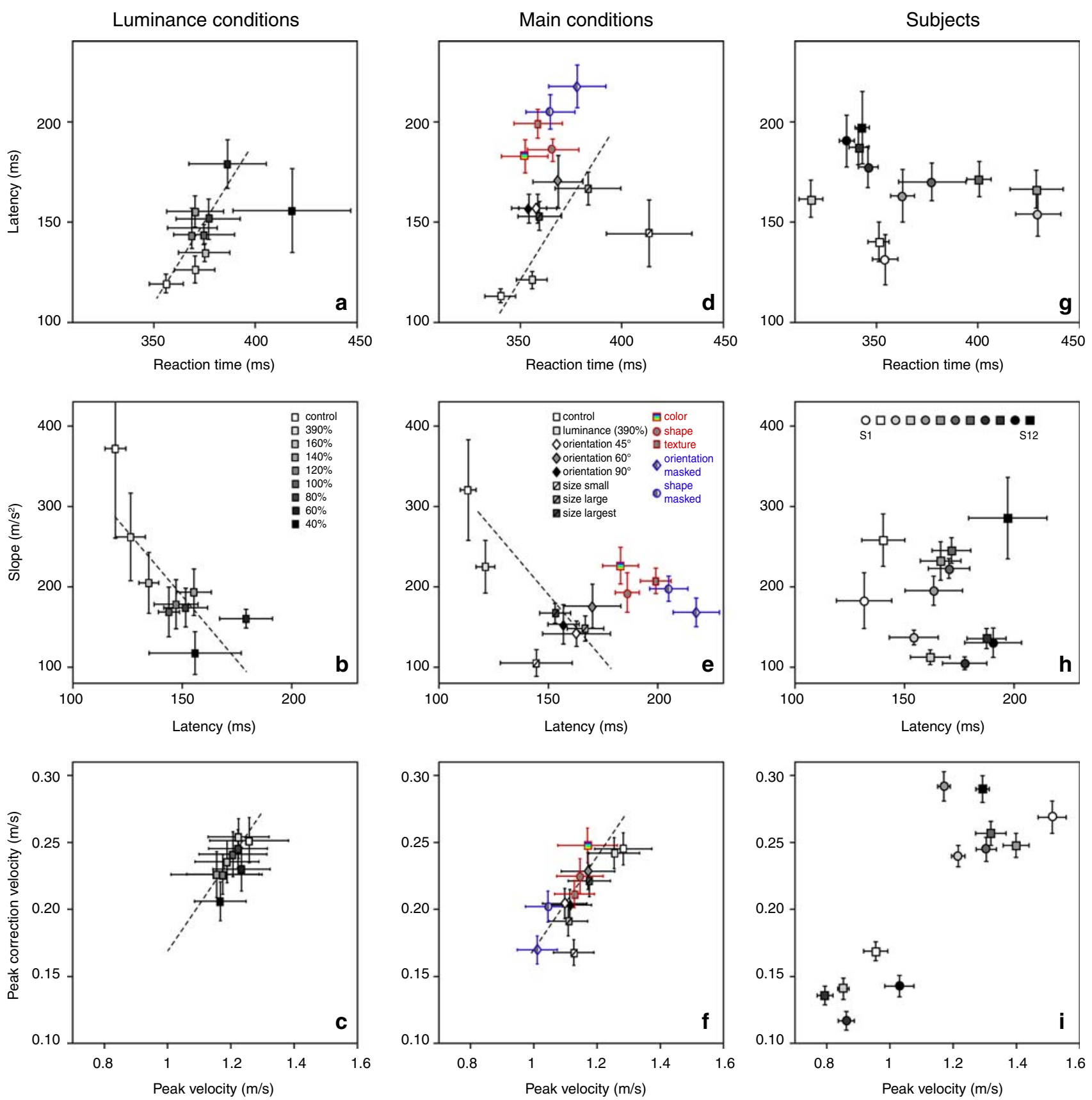

Fig. 4 Comparing the responses across conditions and subjects. a-c Relationship between various movement parameters for the luminance conditions. The latency and slope refer to the correction (Fig. 3). Points are averages across subjects with standard errors. Dashed lines are linear fits through these points. d-f Similar graphs for each of the conditions in which all subjects participated. Symbols in red are the

be due to differences in conspicuousness. Except for the latency (the variable we are interested in), the slope is the variable that varies most clearly with conspicuousness. This is therefore the best measure to use to evaluate whether effects of condition on latency are caused by a difference in conspicuousness, followed by the reaction time (Fig. 4a) and response velocity (Fig. 4c). The correlation of the conditions based on attributes for object recognition; symbols in blue are for conditions in which the change is masked by changing all items. g-i Data for individual subjects, averaged across conditions. The brightness of the symbol indicates the average response latency of the subject (bright is short latency)

conspicuousness with the response velocity (Fig. 4c) is less clear than its correlation with the slope (Fig. 4b). This suggests that a shallower slope of the average response is not only caused by the response being less vigorous, but is also largely caused by more variability in the latency. To verify the effect of variability in latency on the slope of the response, we removed the effect of variability in the latency 
by averaging the responses after synchronizing them at the moment of peak lateral velocity. The responses resulting from this averaging method have almost the same slope for all conditions: $282 \mathrm{~m} / \mathrm{s}^{2}$ with a coefficient of variation of 0.13 (see Fig. 5a). The slopes of the mean additional lateral velocity curves (Fig. 3) are not only much shallower (on average $187 \mathrm{~m} / \mathrm{s}^{2}$ ), but are also much more variable between conditions (coefficient of variation 0.28 ). We conclude that the differences between the slopes of the mean additional lateral velocity curves are mainly caused by differences in the variability in timing between trials (rather than by differences in the intensity of the response). Thus a more conspicuous target results in less variable (Fig. 4b) as well as more vigorous (Fig. 4c) responses.

\section{Different attributes, different latencies?}

Figure $4 \mathrm{~d}$ and e shows the same relationships as Fig. $4 \mathrm{a}, \mathrm{b}$ for the main experimental conditions (Fig. 2a). The dashed lines reproduce the relationship that we found for the luminance conditions (Fig. 4a, b). Participants responded to the various size and orientation conditions with a relationship between latency and conspicuousness that was similar to that for the responses to luminance-defined targets. The targets defined by the attributes color, shape and texture were not less conspicuous than the other targets (reaction times and slopes are similar), but subjects take exceptionally long to react to changes in their position ( $t$-test, $P<0.05)$. These responses were about $50 \mathrm{~ms}$ longer than the ones indicated by the dashed lines. The movements toward the targets defined by color, shape, or texture had similar peak velocities, both of the movements themselves and of the corrections, as the movements toward other targets (Fig. 4f).

For two attributes (orientation and shape) we constructed a "masked" condition, in which not only the target changed location, but also the references changed. This prevents subjects from using the change itself as an indicator of the new target location. The latency for these two conditions is $210 \mathrm{~ms}$, which is longer than for the two corresponding original conditions (paired $t$-test, $P<0.05$ ). The masking decreased the velocity of the movements and of the corrections (Fig. 4f), but did not change the conspicuousness of the targets, either in terms of the reaction time (Fig. $4 \mathrm{~d}$ ) or of the slope of the average response (Fig. 4e). So the increase in latency due to the masking is not caused by masking making the targets less conspicuous, but by requiring additional processing, presumably for a renewed selection of the target. Due to the blocked design, subjects could counteract the effects of their later responses by slowing down their movements (low values for the masked targets in Fig. 4f).

\section{Subjects and amplitudes}

Before discussing how to interpret the results, we want to answer two additional questions. The first is, how does the variability between participants contribute to the results? To find this out, we plotted the data averaged over the conditions for each of the participants separately (Figs. $4 \mathrm{~g}-\mathrm{i}$, $5 b)$. We coded subjects according to the latency of their adjustments: bright curves and symbols correspond to subjects with short latencies. The intensity of the response differed much more between subjects $\left(\mathrm{SD}=91 \mathrm{~m} / \mathrm{s}^{2}\right.$, Fig. $5 b$ ) than between conditions ( $\mathrm{SD}=37 \mathrm{~m} / \mathrm{s}^{2}$, Fig. $5 \mathrm{a}$ ), with no apparent correlation between the peak velocity and latency (Fig. 4i: brighter symbols are not concentrated in one region of the figure). All subjects show more or less the same pattern of latencies as we found for the average data (not shown). This was so for the different attributes as well as for the different levels of conspicuity. There is quite
Conditions

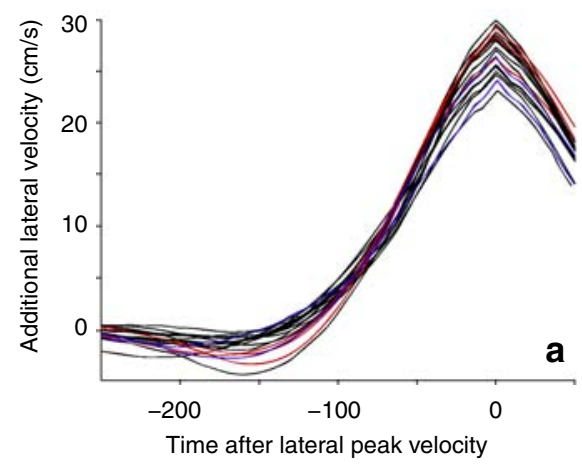

Subjects

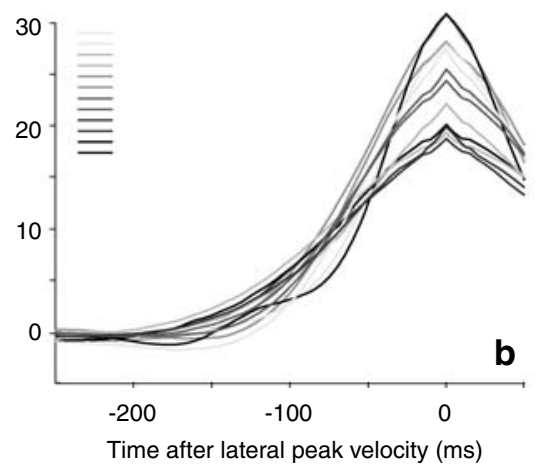

Jump size

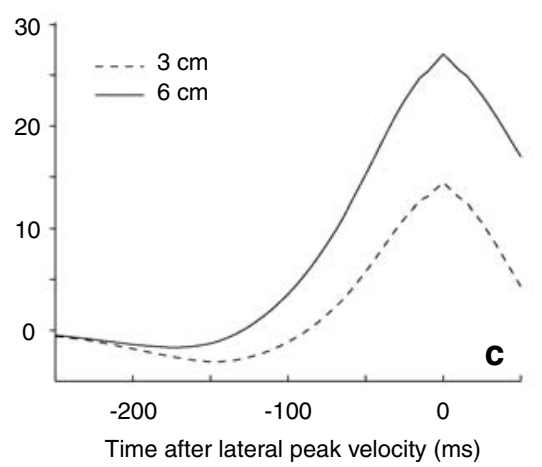

Fig. 5 Magnitudes of the responses as determined by averaging all trials after synchronizing at their peak lateral velocity. a Each curve indicates a condition; color coding as in Fig. 4. The similarity between the curves indicates that the intensity of the response did not differ between conditions. b Responses of subjects. The brightness of the curve indicates the subject's average latency (bright is short latency). c Comparing the responses for the two jump amplitudes. The additional lateral velocity for all trials in which the target jumped $6 \mathrm{~cm}$ was twice as large as that for the trials in which the target jumped $3 \mathrm{~cm}$ 
some variability between the subjects, but subjects that are slow in one condition are generally slow in the other conditions as well. The result is that subjects differ systematically in their latency, without any correlation with their reaction time (Fig. 4g) or with the slope of their response (Fig. 4h). This performance difference cannot easily be explained by factors such as age, visual acuity or body size.

The second question is whether the similarity of the curves in Fig. 5a means that subjects have a pre-programmed response to a perturbation that is used irrespective of the precise visual information. To answer this question, we compared the responses for the two amplitudes of the perturbation. When the target jumped $6 \mathrm{~cm}$ from its initial location (from one extreme position to the other), participants responded about twice as strongly as when the target jumped $3 \mathrm{~cm}$ (Fig. 5c). Thus, the response is proportional to the position change, and therefore clearly tailored to the visual information rather than that pre-programmed and triggered by the change.

\section{Discussion}

By considering the relationship between response latency and measures of conspicuousness (reaction time and variability in the latency), we can conclude that participants reacted with a similar latency to differences in orientation and size than to corresponding differences in luminance. The attributes color, shape and texture gave rise to approximately $50 \mathrm{~ms}$ longer latencies than did differences in luminance, orientation and size that were equally conspicuous. Note that these latencies are still $150 \mathrm{~ms}$ faster than the original reaction times. We found a similar order of fast and more slowly processed attributes across participants, which supports our idea that we can use this method to measure systematic differences between attributes in terms of the time that it takes to process them, even if participants respond differently (see different velocity profiles in Fig. 5b).

An important question in this kind of research is whether the apparent response to a particular attribute could actually be a response to a small difference in another attribute. For instance, in the color condition, participants could respond to small differences in luminance instead of to differences in color between the target and the references, since the luminance of the target and the references was not equated for individual participants. They clearly do not, because if they had done so, the response would be similar to that for one of the small luminance contrast conditions, which is clearly not the case (the slope of the response is much steeper for color).

As mentioned earlier, it takes about as long to respond to changes in position defined by target size as to those defi- ned by target luminance. We cannot reject the possibility that the reactions to the difference in surface area between the target and the references in the size condition were actually responses to the average luminance within an area larger than that of the target itself. To be sure that this was not the case we would have had to vary the luminance of the targets and references, which we did not do in the present study.

Desmurget et al. (1999), showed that applying transcranial magnetic stimulation over the left posterior parietal cortex disrupted corrections of reaching movements of the right hand after a perturbation in target location in four of their five subjects, thereby demonstrating that the dorsal pathway is necessary for fast corrections of the hand. The fact that participants are able to respond very quickly to changes in target location when the target is defined by the attributes orientation, size and luminance (but not color, texture or shape) is therefore an indication for dorsal processing of orientation, size and luminance (but not of color, texture and shape). This division of attributes is consistent with the distinction between pathways for "what" and "where" (Mishkin et al. 1983; Ungerleider and Haxby 1994). The fact that subjects were still able to correct their movements to targets defined by color, texture or shape (albeit at a longer delay) implies that these attributes also reach the parietal cortex, although at a longer latency. This is in line with studies using electrophysiology (Murata et al. 2000; Sakata et al. 2005), positron emission tomography (Faillenot et al. 1997; Vidnyanszky et al. 2000) and functional magnetic resonance imaging (Oliver and ThompsonSchill 2003) that all suggest that the parietal cortex is involved in at least some aspects of discriminating shape. These studies were, however, not concerned with the exact timing of the activity.

Why are responses for targets defined by shape relatively slow? Assuming that all the responses that we measured are indeed mediated by the posterior parietal cortex, the dichotomy in latencies could be explained in two ways. It could be that the parietal cortex mediates the responses to some targets after initial processing of the attributes that are relevant for the "what" of the object (like color, shape and texture (Mishkin et al. 1983) in the ventral pathway. This indirect pathway is likely to result in longer processing times than the direct dorsal pathway for the "where" attributes.

Alternatively, the dichotomy may arise earlier, with the distinction between the magnocellular retinogeniculate pathway that is faster and more sensitive to luminance contrast, but is color-blind and has a low spatial resolution, and the parvocellular pathway that is color selective and has a higher spatial resolution, but is slower and less sensitive to luminance contrast (Livingstone and Hubel 1987, 1988). This distinction corresponds nicely with the dichotomy in 
the latencies that we found. Assuming that both the magnocellular and the parvocellular pathways provide input to the dorsal pathway could therefore account for the different latencies that we found for different attributes (Brenner and Smeets 2003), without requiring input from the ventral pathway.

Although we cannot decide between these two lines of explanation, the two masked conditions indicate what is essential for the very fast responses. Being able to identify the target on the basis of "where" attributes (size, orientation, luminance) is necessary for fast responses. However this is not sufficient, because the latency of responses to the change in location of a target defined by orientation increases by more than $50 \mathrm{~ms}$ if the references change their orientation. Masking changes prevents responses to the location of the change. Presumably, the target has to be found (identified) again, which requires ventral processing (according to the first explanation).

The conclusion that identifying targets by their color takes $50 \mathrm{~ms}$ longer than doing so on the basis of luminance seems to be in conflict with earlier results where we reported that subjects could respond within $120 \mathrm{~ms}$ to color information (Brenner and Smeets 2004). In that study, we compared two conditions: one with one reference object of a different color and one with no reference object (similar to the control condition in the present experiment). The main difference between the responses in these conditions was the larger variation in latency for the condition with one reference object (a more shallow slope). It seems as if the distribution of responses consisted of a small peak at short latency, and a larger peak $50 \mathrm{~ms}$ later (Fig. 3 in Brenner and Smeets 2004). Perhaps, the few very fast responses in the condition with the reference object were related to the very simple design of that experiment: the position to which the target could change was known in advance, so detecting the change in color at the original target location was enough to start an adequate correction. In the present experiment, the location of the new target position had to be determined on the basis of color information. That directly responding to a transient can lead to exceptional fast responses is evident from the fact that masking the jump of the oriented target by orientation changes of the references caused an increase in latency of $50 \mathrm{~ms}$.

A last issue to discuss is the variability between subjects. Some subjects responded consistently later to the perturbations than others (Fig. 4g, h). The difference between the slowest and fastest subject was $70 \mathrm{~ms}$; larger than the difference between the responses to targets defined by color and luminance. Such a large variation is in line with the remarkable finding that stimulating the posterior parietal cortex directly after movement onset disrupts adjustments in some subjects, but not in others (Desmurget et al. 1999). We can draw two conclusions from these variations in latency. The first is that since none of the subjects had particular complaints of clumsiness, the exact value of the latency is apparently not critical for performing every day tasks. The second is that in situations in which short latencies are important, such as when playing table tennis or boxing, some participants may perform better than others could ever hope to, due to differences between their minimal visuomotor latencies. This latter implication (and in particular whether a shorter latency can be achieved by training) is open for experimental verification.

Open Access This article is distributed under the terms of the Creative Commons Attribution Noncommercial License which permits any noncommercial use, distribution, and reproduction in any medium, provided the original author(s) and source are credited.

\section{References}

Brenner E, Smeets JBJ (2003) Perceptual requirements for fast manual responses. Exp Brain Res 153:246-252

Brenner E, Smeets JBJ (2004) Colour vision can contribute to fast corrections of arm movements. Exp Brain Res 158:302-307

Bullier J (2001) Integrated model of visual processing. Brain Res Rev 36:96-107

Cressman EK, Franks IM, Enns JT, Chua R (2006) No automatic pilot for visually guided aiming based on colour. Exp Brain Res 171:174-183

Desmurget M, Epstein CM, Turner RS, Prablanc C, Alexander GE, Grafton ST (1999) Role of the posterior parietal cortex in updating reaching movements to a visual target. Nat Neurosci 2:563-567

DeYoe EA, Van Essen DC (1988) Concurrent processing streams in monkey visual-cortex. Trends Neurosci 11:219-226

Faillenot I, Toni I, Decety J, Gregoire MC, Jeannerod M (1997) Visual pathways for object-oriented action and object recognition: functional anatomy with PET. Cereb Cortex 7:77-85

Goodale MA, Milner AD (1992) Separate visual pathways for perception and action. Trends Neurosci 15:20-25

Lee JH, van Donkelaar P (2002) Dorsal and ventral visual stream contributions to perception-action interactions during pointing. Exp Brain Res 143:440-446

Livingstone MS, Hubel DH (1987) Psychophysical evidence for separate channels for the perception of form, color, movement, and depth. J Neurosci 7:3416-3468

Livingstone M, Hubel D (1988) Segregation of form, color, movement, and depth: anatomy, physiology, and perception. Science 240:740-749

Mishkin M, Ungerleider LG, Macko KA (1983) Object vision and spatial vision: two cortical pathways. Trends Neurosci 6:414417

Murata A, Gallese V, Luppino G, Kaseda M, Sakata H (2000) Selectivity for the shape, size, and orientation of objects for grasping in neurons of monkey parietal area AIP. J Neurophysiol $83: 2580-2601$

Oliver RT, Thompson-Schill SL (2003) Dorsal stream activation during retrieval of object size and shape. Cogn Affect Behav Neurosci 3:309-322

Pisella L, Arzi M, Rossetti Y (1998) The timing of color and location processing in the motor context. Exp Brain Res 121:270-276

Rossetti Y, Pisella L, Pélisson D (2000) New insights on eye blindness and hand sight: temporal constraints of visuo-motor networks. Vis Cogn 7:785-808 
Sakata H, Tsutsui KI, Taira M (2005) Toward an understanding of the neural processing for 3D shape perception. Neuropsychologia 43:151-161

Schmidt T (2002) The finger in flight: real-time motor control by visually masked color stimuli. Psychol Sci 13:112-118

Schmolesky MT, Wang YC, Hanes DP, Thompson KG, Leutgeb S, Schall JD, Leventhal AG (1998) Signal timing across the macaque visual system. J Neurophysiol 79:3272-3278

Tanne J, Boussaoud D, BoyerZeller N, Rouiller EM (1995) Direct visual pathways for reaching movements in the macaque monkey. Neuroreport 7:267-272
Trevarthen CB (1968) Two mechanisms of vision in primates. Psychol Forsch 31:299-348

Ungerleider LG, Haxby JV (1994) 'What' and 'where' in the human brain. Curr Opin Neurobiol 4:157-165

Vidnyanszky Z, Gulyas B, Roland PE (2000) Visual exploration of form and position with identical stimuli: functional anatomy with PET. Hum Brain Mapp 11:104-116

White BJ, Kerzel D, Gegenfurtner KR (2006) Visually guided movements to color targets. Exp Brain Res 175:110-126 